\title{
Differences in subcortical structures in young adolescents at familial risk for schizophrenia: a preliminary study
}

\author{
Michael Doughertya ${ }^{\mathrm{*}}$, Hongbin Gua ${ }^{\mathrm{a}}$, Joshua Bizzella,b, Stacy Ramseya ${ }^{\mathrm{a}}$, Guido Gerig ${ }^{\mathrm{c}}$, Diana \\ Perkins $^{\mathrm{a}}$, and Aysenil Belger ${ }^{\mathrm{a}, \mathrm{b}, \mathrm{d}}$ \\ aUniversity of North Carolina School of Medicine, Department of Psychiatry, Chapel Hill, North \\ Carolina, United States \\ ${ }^{b}$ Duke-UNC Brain Imaging and Analysis Center, Durham, North Carolina, United States \\ cUniversity of Utah, Scientific Computing and Imaging Institute, Salt Lake City, Utah, United \\ States \\ dNeurodevelopmental Disorders Research Center, University of North Carolina School of \\ Medicine, Chapel Hill, North Carolina, United States
}

\begin{abstract}
Schizophrenia has been associated with reduced volumes of subcortical structures on MRI, but the relation of these reductions to familial risk for the disorder is unclear. We investigated the effect of familial risk for schizophrenia on regional subcortical volumes during adolescence, a period marked by steep maturational changes in brain structure and the emergence of psychotic symptoms. A group of 26 non-help-seeking, first-degree relatives of patients with schizophrenia and 43 matched healthy comparisons, between 9 and 18 years of age, underwent MRI scanning and were rated for the presence of prodromal symptoms. Five subcortical regions-of-interest were tested for group differences and group by age interactions, as well as correlations with low-level prodromal symptoms in the familial risk group. Relative to comparisons, familial risk subjects demonstrated greater positive volume-age relationships in hippocampus, putamen, and globus pallidus. These results suggest that relatives of individuals with schizophrenia exhibit structural abnormalities in the subcortex as early as pre-adolescence, which may reflect altered neurodevelopment of these regions.
\end{abstract}

\section{Keywords}

hippocampus; putamen; basal ganglia; neurodevelopment; MRI; volumetric

\section{Introduction}

Although the pathophysiological mechanisms underlying schizophrenia remain unknown, the disorder has long been associated with alterations in brain structure (Shenton et al.,

\footnotetext{
(C) 2012 Elsevier Ireland Ltd. All rights reserved.

Corresponding Author: Michael Dougherty, at the University of North Carolina School of Medicine, Department of Psychiatry; "Michael.Dougherty@uphs.upenn.edu; 250 Medical School Building D, CB \# 7160, Chapel Hill, NC, 27599-7160; Phone: (919) 368-5918; Fax: (919) 966-8004.

Publisher's Disclaimer: This is a PDF file of an unedited manuscript that has been accepted for publication. As a service to our customers we are providing this early version of the manuscript. The manuscript will undergo copyediting, typesetting, and review of the resulting proof before it is published in its final citable form. Please note that during the production process errors may be discovered which could affect the content, and all legal disclaimers that apply to the journal pertain.
} 
2001). Volumetric differences from healthy comparisons have been reported as early as the first episode of psychosis (Ellison-Wright et al., 2008; Vita et al., 2006), in adolescents with schizophrenia (Rapoport and Gogtay, 2011), in prodromal individuals, and in unaffected relatives (Boos et al., 2007; Fusar-Poli et al., 2011). Few studies, however, have examined asymptomatic relatives during early adolescence, a stage preceding the period of greatest psychosis risk. Studies of brain maturation in children and adolescents with familial risk for schizophrenia are challenged by the difficulty of controlling for normal developmental effects during a period of rapid cerebral change. It is nevertheless likely that the dynamic functional and structural brain changes that occur during puberty and the early adolescent period represent in and of themselves a factor increasing vulnerability for neuropsychiatric disorders (Paus et al., 2008). Adolescence therefore constitutes a critical period for targeted investigations into the pathogenesis of schizophrenia.

Among the many brain regions that are particularly promising candidates for investigation in the neurodevelopmental hypothesis of schizophrenia are the subcortical regions of the medial temporal lobe (MTL) and the basal ganglia (BG). The hippocampus is an MTL structure that is among the most studied regions in schizophrenia research, and there is now a general consensus that at least some high-risk (HR) subjects experience hippocampal reduction prior to psychosis onset (Boos et al., 2007; Fusar-Poli et al., 2011). The trend during this time period is complex however, with separate studies supporting environmental (Lawrie et al., 2001; Mattai et al., 2011) and hereditary (Goldman et al., 2008) factors as the dominant force behind pre-psychotic hippocampal reduction. Furthermore, the hippocampus and its MTL counterpart the amygdala may be variably affected depending on the presence of an affective component of psychosis (Velakoulis et al., 2006), and comparisons across studies of MTL structures are made more difficult by methodological differences, such as the practice in many older studies of examining the amygdala and hippocampus as one complex.

The comparatively fewer studies on the BG in schizophrenia support abnormalities of these regions, with extensive reports of functional differences from controls in caudate, putamen, and globus pallidus (Hazlett et al., 2008; Hoffman et al., 2011; Li et al., 2010; Menon et al., 2001; Morey et al., 2005). Although the BG are difficult to study because they are known to be among the most sensitive regions of the brain to effects of antipsychotics (Brandt and Bonelli, 2008; Ellison-Wright et al., 2008), the functional literature has recognized a significant role of hypermetabolic activity in these regions even in prodromal and unmedicated physiology (Fusar-Poli et al., 2010; Huang et al., 2010; Kegeles et al., 2010; Müller et al., 2002). While there is evidence supporting a correlation of such hypermetabolism with decreased caudate volume (Jayakumar et al., 2006), there is still no consensus on the structural correlate of these prepsychotic and unmedicated functional differences. Caudate volumes have generally been found to be smaller at disease onset (Brandt and Bonelli, 2008; Ellison-Wright et al., 2008), whereas reductions in the lentiform nuclei have been less frequently reported (Ballmaier et al., 2008). Volumetric studies of unaffected relatives are conflicting however (Goldman et al., 2008; Lawrie et al., 2001; Rajarethinam et al., 2007), leaving the contribution of genetic risk in BG uncertain.

By defining how cohorts of familial high risk (FHR) individuals differ from comparable individuals in the general population, especially during an age range that has been relatively understudied and in brain structures whose exact role in pathogenesis is still unclear, the current study aimed to characterize possible pre-symptomatic alterations in neurodevelopment at the level of regional subcortical morphology. Any detected abnormality may or may not prove to be a predictor of conversion on later follow-up. We examined volumetric differences in the regions of the hippocampus, amygdala, putamen, globus pallidus, and caudate nucleus, in non-help-seeking child and adolescent relatives of 
schizophrenia patients versus healthy comparisons. We further examined whether observed differences were modulated by age, reflecting possible alterations in adolescent neurodevelopment. Finally, we also explored the correlation between regional volumes and the severity of any prodromal psychotic symptoms in the HR group. We hypothesized that FHR adolescents would display volumetric reductions in the subcortical regions, that differences would be greatest in older individuals (presumed closest to possible conversion), and that these regional volumes would negatively correlate with the severity of their subsyndromal clinical symptomatology.

\section{Methods}

\subsection{Subjects}

The current study presents the baseline structural MRI data for the first sample recruited in an ongoing multimodal, longitudinal study of children and adolescents with FHR for schizophrenia by the University of North Carolina (UNC) Conte Center. Identification and recruitment of 26 FHR subjects between ages 9-18 years was done through the specialized schizophrenia treatment services of UNC hospitals and affiliated clinics, as well as through consumer organizations (North Carolina National Alliance on Mental Illness and Mental Health Association). Healthy comparison $(\mathrm{HC})$ subjects $(n=43)$ were recruited from the same local communities as the FHR subjects, via email advertisements to databases of UNC students and employees as well as county public schools. Subjects were matched for sex, age, and ethnicity. In order to yield a relatively continuous distribution of subjects in each group across the age range, recruitment was structured into three age brackets: 9-11, 12-14 and $15-18$ years old.

For all subjects, FHR was defined as family history of schizophrenia or schizoaffective disorder in a first-degree relative, and was confirmed with the Family Interview for Genetic Studies (Maxwell, 1996). Diagnosis of the affected relative was confirmed using either the Structured Clinical Interview for DSM IV disorders (adults) or the Washington University Kiddie Schedule for Affective Disorders and Schizophrenia (WASH-U-KSADS) (children) (Geller, 1996). All subjects had a WASH-U-KSADS. Any Axis I disorder in a healthy comparison or their first-degree relatives resulted in exclusion; FHR subjects meeting criteria for a psychotic disorder or bipolar disorder were excluded. Subjects were also excluded if they had a serious medical or neurological disorder, or a history of antipsychotic treatment within three months of enrollment. All included subjects underwent a baseline clinical evaluation for the presence and severity of positive, negative, disorganized, and general symptoms, and scores were assigned on each dimension using the Scale of Prodromal Symptoms (SOPS) (Miller et al., 1999). None of the FHR subjects were treatment-seeking at the time of recruitment into the study.

Subjects under 18 years of age gave written assent to participation in the study, while legal guardians of minors and 18-year-old subjects provided written informed consent. The UNC Biomedical Institutional Review Board approved the study.

\subsection{Image acquisition and analysis}

All subjects were scanned on a $3 \mathrm{~T}$ General Electric short-bore scanner at the Duke-UNC Brain Imaging Analysis Center. Multi-contrast high resolution MRI pulse sequences were used to allow multi-channel segmentation for optimal fidelity, including T1 weighting (IRprepped 3-D FSPGR, TR $7.5 \mathrm{~ms}$, TE $3.0 \mathrm{~ms}$, inversion preparation time $450 \mathrm{~ms}$, flip angle $12^{\circ}$, bandwidth/pixel $244 \mathrm{~Hz}$, imaging matrix $256 \times 256$, FOV $256 \times 256 \mathrm{~mm}$, slice thickness $1 \mathrm{~mm}$ ) and a double-echo dual-contrast FSE sequence (TR 3000) ms, TE 25.1 and $87.7 \mathrm{~ms}$, flip angle $90^{\circ}$, bandwidth/pixel $122.1 \mathrm{~Hz}$, imaging matrix $256 \times 192$, FOV $256 \times$ 
$256 \mathrm{~mm}$, slice thickness $2 \mathrm{~mm}$ ) for optimized proton density and T2 weighting. Total brain and tissue volumes were obtained with an automatic, expectation-maximization scheme (EMS) brain segmentation tool which used all three MRI contrasts and an atlas prior (Prastawa et al., 2003). A pipeline for automated subcortical segmentation, developed by investigators at the Neuro Image Research and Analysis Laboratories (NIRAL) at UNC and based on an unbiased population atlas embedding probabilistic models of anatomical structures (Gouttard et al., 2007), was used to delineate the subcortical regions-of-interest (ROI). Each image was visually inspected for gross segmentation errors by two blinded raters, and no such errors were detected. In the interest of preserving the objectivity of the automated method, we elected to neither exclude nor manually correct the frequent but minor errors in boundaries produced by the automated, probabilistic algorithm.

\subsection{Statistical analyses}

Demographic variables were analyzed with the Fischer Exact Test for categorical variables and the Wilcoxon Two-Sample Rank Test for continuous variables. The categorical variables included sex, race, handedness, and highest level of parental education, which was used as a rough proxy for socioeconomic status and home environment. The continuous variables analyzed were each subject's age and SOPS scores.

The group and age related differences in total brain tissue volume (TBV=total gray matter $[\mathrm{GM}]+$ total white matter [WM]) were first examined in an ANCOVA model with group, sex, age, and group by age interaction. The five subcortical ROI were then normalized by dividing each by the TBV to correct for individual differences in head size (corrected volume $=\mathrm{ROI}$ volume/TBV $* 100,000$ ). Mean raw ROI volumes are listed in a supplementary table, and for the remainder of the manuscript, ROI "volume" will refer to "TBV-corrected volume" unless otherwise specified. We tested for group differences in hemispheric asymmetry by group X hemisphere interaction in a MANCOVA model with group (FHR, HC), age (9-18 years), and sex (male, female) as between-subject variables and hemisphere (left, right) as a within-subject variable. With no group $\mathrm{X}$ hemisphere interactions detected, we combined corresponding left and right subcortical structures and modeled the total volume with ANCOVA models, which include group, sex, and age as well as group by age interaction. Quadratic age trend was tested but dropped from the models due to nonsignificant findings and limited sample size. The volumetric differences associated with age were estimated by separate age slopes for the FHR and HC groups. Group differences in the volume-age relationship were tested in the interaction between age and group.

Within the high-risk group, Pearson's $r$ was used to test the association of each SOPS domain (positive, negative, disorganized, general, and total scores) with corrected volume of each subcortical ROI. As a sensitivity analysis to evaluate the potential confounding effect of age, we calculated the partial correlation between SOPS scores and ROI volume while controlling for age.

All tests were two-tailed at significance level of 0.05 . To further safeguard against Type I error, we corrected for multiple comparisons using the false discovery rate (FDR) adjustment.

\section{Results}

\subsection{Demographics}

The sociodemographic profile of the sample is shown in Table 1. The familial risk group did not differ significantly from the comparison group in age, sex, ethnicity, handedness, or last grade completed. Parents of the HC group had significantly higher levels of education than those of the FHR group $(P<0.01)$, and HC subjects also differed from FHR by the absence of 
Axis I diagnoses. Even using the conservative, non-parametric Wilcoxon test, SOPS scores were significantly higher in the FHR group in all dimensions, although the scores still fell well below a level that could be considered "prodromal" (Miller et al., 1999).

\subsection{Volumetric analyses}

3.2.1. Total Brain Volume-The ANCOVA model of TBV (Table 2) demonstrated a nearly-significant trend toward larger total volume in the male adolescents than the females, after FDR adjustment. The FHR and HC groups had similar TBV overall and in relation to age, with both study groups showing modest annualized differences of less than $1 \%$ in TBV (Figure 1). In the post hoc analysis of the slope of TBV-age relationship, the increase was significant in the $\mathrm{HC}$ group.

3.2.2. Hemisphere asymmetry-MANCOVA models found significant hemispheric asymmetry in all subcortical structures $(P<0.01)$, but no significant differences between groups $(P>0.50)$. For both FHR and HC, amygdala and caudate were about $3 \%$ larger on the right, while hippocampus, putamen, and globus pallidus were larger on the left by $5 \%, 2 \%$ and $1 \%$ respectively (Supplementary Table 2).

3.2.3. Subcortical structures-With no evidence for group differences in hemispheric asymmetry, data for each ROI was collapsed across both hemispheres to test for group- and age-related differences (Table 2). Effect of sex was not significant in any ROI after FDR adjustment. Averaging across the age range, the FHR group showed smaller volumes than the $\mathrm{HC}$ group in all the subcortical structures. This difference was initially significant in the hippocampus, but did not survive FDR correction. In the analysis of group differences in age effects however, the groups showed significantly different volume-age relationships in most subcortical structures $(P=0.10$ for amygdala, $P=0.06$ in caudate, and $P<0.05$ for hippocampus, putamen, and globus pallidus with FDR correction; see Table 2 and Figure 1). Post hoc analysis of each group revealed that the TBV-corrected volumes were largely stable across adolescence in the HC group, with non-significant $(P>0.18)$ slopes in all ROIs. In contrast, significant positive-sloping volume-age relationships $(P<0.05)$ were observed in all subcortical structures, relative to TBV, for the FHR group (Table 2 and Figure 1).

\subsection{Correlations with Prodromal Symptoms}

Table 3 shows Pearson's $r$ with corresponding correlations between subcortical structures and SOPS symptoms. None of the correlations were significant after FDR adjustment, although the greatest trends were toward a negative correlation between ROI volume and disorganization scores ( $r=-0.58$ to $-0.60, P=0.13$ for hippocampus and putamen). The sensitivity analysis controlling for age did not change the significance of any results (see Supplementary Table 3).

\section{Discussion}

We found that relative to healthy comparisons, FHR subjects displayed significant group by age interactions such that TBV-corrected subcortical volumes were larger with age. Volumes were smaller for FHR's in all ROI's, suggesting a trend of initially decreased volumes which approach normal during adolescence.

This study's cohort is unique in that subjects span the entire range of adolescence, including early adolescence. Individuals who go on to develop schizophrenia begin to exhibit neuropsychological abnormalities as early as childhood (Jones et al., 1994), and dramatic neurodevelopmental shifts during adolescence may be critical precipitants of a spectrum of psychopathology (Paus et al., 2008). Therefore, in order to characterize biological markers 
of risk for schizophrenia in an early presymptomatic stage, the current study encompassed the entire range of adolescence in a non-prodromal FHR cohort.

Our data are consistent with well-established hippocampal reductions in FHR individuals (Boos et al., 2007; Fusar-Poli et al., 2011), although the overall group effect did not survive FDR adjustment. It should be noted that while not reaching significance, other subcortical structures all showed reductions in the same direction. The main findings of our study, namely the positivesloping group $\mathrm{X}$ age interactions of the FHR group, were less anticipated. Regarding the hippocampus, the size of this structure has been shown to exhibit little change relative to intracranial volume (ICV) during healthy adolescence (Mattai et al., 2011; Ostby et al., 2009), as was the case in our healthy comparisons. Among FHR studies, a recent comparison of healthy subjects and unaffected siblings of childhood-onset schizophrenia (COS) patients failed to find a significant difference in volume-age relationship, in the setting of non-significant, downwardsloping volume-age curves in both groups (Mattai et al., 2011). The trajectories of Mattai and colleague's sample could be at least partially consistent with ours however, in that non-significant differences between groups are greatest at younger ages. The lack of significance may be due to qualitative differences between our cohort and that of Mattai et al, which included only siblings of childhood-onset patients who were also free of any lifetime Axis I disorder (rather than only psychotic disorders). Interestingly, our study replicates the previously isolated report of positive-sloping group $\mathrm{X}$ age interactions in the hippocampi of slightly older FHR's with some incidence of non-psychotic mental illness (Ho and Magnotta, 2010).

Despite this isolated report in the hippocampus, positive volume-age relationships in the subcortex have little precedent in unmedicated subjects. Longitudinal studies of pediatric neurodevelopment at the National Institutes of Mental Health have revealed that in healthy adolescents the caudate nucleus appears to follow an "inverted U" trajectory across adolescence (Lenroot et al., 2007). Our study's ability to detect second-order age effects was likely limited by sample size, but our HC plots seem compatible with the subtle negative slope of the post-apical portion of the NIMH plots corresponding to our cohort's age range. Other subcortical trajectories are less well-studied, but the lenticular nuclei may follow a similar, declining trajectory (Giedd et al., 1996; Ostby et al., 2009), while the amygdala, much like the hippocampus, may also normally exhibit a relatively flat growth trajectory (Giedd et al., 1999). Considering the general absence of literature on subcortical structures in early adolescent FHR cohorts, the trajectories found in our study could possibly provide a novel framework for interpreting the FHR literature across larger age ranges. For instance, an adolescent study found smaller caudates in FHR's (Rajarethinam et al., 2007), but studies of unaffected adult relatives have more frequently failed to detect such reductions (Goldman et al., 2008; Hannan et al., 2010; Mamah et al., 2008). These results would be consistent with a volumetric convergence of groups with age.

The possible explanations for our age-related findings are several. Due to the cross-sectional nature of our sample, we cannot determine whether the group $\mathrm{X}$ age interactions mirror true intra-subject changes. If cerebral change corresponding to our volume-age relationships could be demonstrated in a longitudinal design, it would be the first example in the subcortex of FHR subjects of the principle that trajectories of cerebral development may be unique markers of psychopathology during adolescence (Shaw et al., 2010). This principle has been demonstrated in the striatum of developmental cohorts with autism (Langen et al., 2009) and ADHD (Castellanos et al., 2002), as well as the cortex of early-onset schizophrenia (Rapoport and Gogtay, 2011). An increase in corrected subcortical volumes with age could also be a surrogate marker of pathology, considering that TBV-correction allows for the possibility that our group $\mathrm{X}$ age interactions reflect more of a diminution in other cerebral structures rather than a disproportionate increase in the studied ROI's. 
Alternate compartments that could be exhibiting such a divergence from the HC trajectory would include cortical GM or WM. The cerebellum is a third, underappreciated compartment that seems to exhibit a compatible negative volume-age relationship in FHR adolescents both in post-hoc, informal analyses of our raw data as well as a recent report of a similar cohort (Greenstein et al., 2011).

Follow-up data from our study's cohort may reveal clues as to whether the FHR group's age-related differences in relative subcortical volumes represent a suboptimal adaptation to a premorbid process, serving as a marker for persistently elevated risk, or instead an effective compensation that is protective against psychosis. Considering the established findings of reduced corrected volumes of several of the subcortical ROI's in unmedicated first-episode patients, it seems more likely that our volume-age relationships are modeling some type of compensatory or "normalizing" process for an intrinsic vulnerability phenotype. Only a minority of FHR individuals (6-21\% depending on type) will actually go on to develop psychosis (Kendler et al., 1993). This is consistent with our sample that is characterized by low (within normal limits) SOPS scores, a conversion rate of 1/26, and no greater than 5/26 separate subjects demonstrating potentially "help-seeking" behaviors after 1-4 years of follow-up (data available upon request). The concept of volumetric change mirroring clinical outcome has a precedent in other neuroanatomical regions in childhood psychoses, as well as other psychiatric disorders (Shaw et al., 2010). While cerebral plasticity during the adolescent period may provide a setting in vulnerable individuals for the emergence of psychiatric symptoms in response to "triggers" such as psychological stress and substance abuse (Lodge and Grace, 2011; Paus et al., 2008; Rapoport and Gogtay, 2008), such plasticity may also allow for restitutive mechanisms in the absence of such triggers. The positive volume-age relationships in this study could theoretically represent such adaptive maturational mechanisms in the subcortex.

As previously alluded to, we must also consider the limitations of a cross-sectional approach in interpretations of group X age interactions. Specifically, cohort effects could produce interactions in our sample which in reality represent inter- rather than intra-subject differences by age. This could occur by chance due to our relatively small sample size, but also could be a product of our study criteria. The natural history of schizophrenia suggests that a certain percentage of young, non-help-seeking FHR individuals will develop symptoms or even convert before age 18, which would result in exclusion from this study. In other words, it is possible that older FHR individuals in our study represent a slightly lower risk group by virtue of having moved later in the risk-age window without becoming symptomatic.

The exploratory correlation analyses initially revealed negative correlations between disorganized symptoms and volumes of amygdala, hippocampus, and putamen. These findings are consistent with previous reports suggesting that the disorganization domain may index many of the cognitive deficits of the schizophrenia spectrum (Demjaha et al., 2010). Such deficits are among the earliest symptomatic manifestations of disease and/or disease risk (Jones et al., 1994) and also associated with diminished subcortical volumes in high-risk samples (Bhojraj et al., 2011; Hannan et al., 2010; van Erp et al., 2008). Our results did not survive FDR adjustment and thus should be interpreted cautiously, especially in light of the potential non-specificity of disorganization symptoms. Future studies should attempt to explore these trends with larger sample sizes.

Study limitations not already mentioned include the potential for the somewhat broad delineations of ROI's to mask more striking effects at the subregional level, for example in anterior hippocampus or head of the caudate. Evaluation of other relevant ROI's, such as the thalamus and nucleus accumbens, could have provided additional insight into abnormalities 
of subcortical circuitry in FHR subjects, but software limitations prevented us from reliably examining these structures. Sample size was small for a cross-sectional study looking at brain structure during such a dynamic period of development, which will also be confounded by gender differences and the onset of puberty. Interactions of sex with age and group should be examined in future studies with larger sample sizes, in conjunction with pubertal markers such as Tanner stage or gonadal hormone levels.

Our sample groups were relatively well-matched except for a significant difference in maximum degree of parental education. This is a common hazard of FHR studies, in that by definition a much larger portion of FHR subjects have parents with persistent mental illness, a characteristic significantly associated with lower educational status. We were unable to disentangle these factors in our model, and are therefore unable to discount the potential role of familial environment compared to that of heredity in our findings. Additionally, while significant genetic overlap exists between the two conditions (Kendler et al., 1993; Tienari et al., 2003), our FHR subjects cannot be strictly classified as only susceptible to schizophrenia, as the sample also includes relatives of patients with schizoaffective disorder.

In summary, we have demonstrated abnormalities of hippocampal and basal ganglia volume-age relationships in a sample of non-prodromal adolescents with familial risk for schizophrenia. The findings add to the body of literature suggesting that the hippocampus and basal ganglia may be already developing aberrantly prior to the onset of psychosis in high-risk individuals, and therefore related to illness vulnerability. This study is a valuable exploratory investigation of a cohort traditionally difficult to analyze, and the first to our knowledge to examine these particular regional trajectories in relatives of patients with schizophrenia during the dynamic period of adolescent development from 9-18 years of age. Replication in a longitudinal design as well as correlation with conversion status is necessary to confirm and expand the significance of these findings. This will contribute to an enhanced understanding of the pre-symptomatic period, which may aid in the identification of "triggers" of psychosis and the development of targeted, early interventions.

\section{Supplementary Material}

Refer to Web version on PubMed Central for supplementary material.

\section{Acknowledgments}

This study was supported by the National Institutes of Health (Silvio O. Conte Center MH064065) and National Alliance for Medical Image Computing (U54 EB005149), as well as the Howard Holderness Distinguished Medical Scholars Fellowship (to M.D.), and NIH (2-R01MH058251), Foundation of Hope, and National Alliance for Research on Schizophrenia and Depression Independent Investigator Award (to A.B.). We would also like to thank Kathleen Monforton and Erin Douglas for subject recruitment, Susan Music, Zoe Englander, Michael Casp, and Mary Agnes McMahon for image acquisition, Joe Blocher for image pre-processing, and Robert Hamer for manuscript advice.

\section{References}

Ballmaier M, Schlagenhauf F, Toga AW, Gallinat J, Koslowski M, Zoli M, Hojatkashani C, Narr KL, Heinz A. Regional patterns and clinical correlates of basal ganglia morphology in non-medicated schizophrenia. Schizophrenia Research. 2008; 106:140-147. [PubMed: 18818054]

Bhojraj TS, Francis AN, Montrose DM, Keshavan MS. Grey matter and cognitive deficits in young relatives of schizophrenia patients. NeuroImage. 2011; 54:S287-S292. [PubMed: 20362681]

Boos HBM, Aleman A, Cahn W, Pol HH, Kahn RS. Brain Volumes in Relatives of Patients With Schizophrenia: A Meta-analysis. Arch Gen Psychiatry. 2007; 64:297-304. [PubMed: 17339518]

Brandt GN, Bonelli RM. Structural neuroimaging of the basal ganglia in schizophrenic patients: a review. WMW Wiener Medizinische Wochenschrift. 2008; 158:84-90. 
Castellanos FX, Lee PP, Sharp W, Jeffries NO, Greenstein DK, Clasen LS, Blumenthal JD, James RS, Ebens CL, Walter JM, Zijdenbos A, Evans AC, Giedd JN, Rapoport JL. Developmental Trajectories of Brain Volume Abnormalities in Children and Adolescents With Attention-Deficit/Hyperactivity Disorder. JAMA: The Journal of the American Medical Association. 2002; 288:1740-1748. [PubMed: 12365958]

Demjaha A, Valmaggia L, Stahl D, Byrne M, McGuire P. Disorganization/Cognitive and Negative Symptom Dimensions in the At-Risk Mental State Predict Subsequent Transition to Psychosis. Schizophrenia Bulletin [Epub ahead of print]. 2010

Ellison-Wright I, Glahn DC, Laird AR, Thelen SM, Bullmore E. The Anatomy of First-Episode and Chronic Schizophrenia: An Anatomical Likelihood Estimation Meta-Analysis. Am J Psychiatry. 2008; 165:1015-1023. [PubMed: 18381902]

Fusar-Poli P, Borgwardt S, Crescini A, Deste G, Kempton MJ, Lawrie S, Mc Guire P, Sacchetti E. Neuroanatomy of vulnerability to psychosis: A voxel-based meta-analysis. Neuroscience \& Biobehavioral Reviews. 2011; 35:1175-1185. [PubMed: 21168439]

Fusar-Poli P, Howes OD, Allen P, Broome M, Valli I, Asselin M-C, Grasby PM, McGuire PK. Abnormal Frontostriatal Interactions in People With Prodromal Signs of Psychosis: A Multimodal Imaging Study. Arch Gen Psychiatry. 2010; 67:683-691. [PubMed: 20603449]

Geller, B.; Zimerman, B.; Williams, M.; Frazier, J. WASH-U-KSADS (Washington University at St. Louis Kiddie and Young Adult Schedule for Affective Disorders and Schizophrenia - Lifetime and Present Episode Version for DSM-IV). St. Louis, MO: Washington University School of Medicine; 1996.

Giedd JN, Jeffries NO, Blumenthal J, Castellanos FX, Vaituzis AC, Fernandez T, Hamburger SD, Liu H, Nelson J, Bedwell J, Tran L, Lenane M, Nicolson R, Rapoport JL. Childhood-onset schizophrenia: progressive brain changes during adolescence. Biological Psychiatry. 1999; 46:892-898. [PubMed: 10509172]

Giedd JN, Snell JW, Lange N, Rajapakse JC, Casey BJ, Kozuch PL, Vaituzis AC, Vauss YC, Hamburger SD, Kaysen D, Rapoport JL. Quantitative Magnetic Resonance Imaging of Human Brain Development: Ages 4-18. Cerebral Cortex. 1996; 6:551-559. [PubMed: 8670681]

Goldman AL, Pezawas L, Mattay VS, Fischl B, Verchinski BA, Zoltick B, Weinberger DR, MeyerLindenberg A. Heritability of Brain Morphology Related to Schizophrenia: A Large-Scale Automated Magnetic Resonance Imaging Segmentation Study. Biological Psychiatry. 2008; 63:475-483. [PubMed: 17727823]

Gouttard, S.; Styner, M.; Joshi, S.; Smith, RG.; Hazlett, HC.; Gerig, G. Subcortical structure segmentation using probabilistic atlas priors, Medical Imaging 2007: Image Processing. 1 ed. San Diego, CA, USA: SPIE; 2007. 65122J-65111

Greenstein D, Lenroot R, Clausen L, Chavez A, Vaituzis AC, Tran L, Gogtay N, Rapoport J. Cerebellar development in childhood onset schizophrenia and non-psychotic siblings. Psychiatry Research: Neuroimaging. 2011; 193:131-137.

Hannan KL, Wood SJ, Yung AR, Velakoulis D, Phillips LJ, Soulsby B, Berger G, McGorry PD, Pantelis C. Caudate nucleus volume in individuals at ultra-high risk of psychosis: A crosssectional magnetic resonance imaging study. Psychiatry Research: Neuroimaging. 2010; 182:223230.

Hazlett EA, Buchsbaum MS, Zhang J, Newmark RE, Glanton CF, Zelmanova Y, Haznedar MM, Chu K-W, Nenadic I, Kemether EM, Tang CY, New AS, Siever LJ. Frontal-striatal-thalamic mediodorsal nucleus dysfunction in schizophrenia-spectrum patients during sensorimotor gating. NeuroImage. 2008; 42:1164-1177. [PubMed: 18588988]

Ho B-C, Magnotta V. Hippocampal volume deficits and shape deformities in young biological relatives of schizophrenia probands. NeuroImage. 2010; 49:3385-3393. [PubMed: 19941961]

Hoffman RE, Fernandez T, Pittman B, Hampson M. Elevated Functional Connectivity Along a Corticostriatal Loop and the Mechanism of Auditory/Verbal Hallucinations in Patients with Schizophrenia. Biological Psychiatry. 2011; 69:407-414. [PubMed: 21145042]

Huang X-Q, Lui S, Deng W, Chan RCK, Wu Q-Z, Jiang L-J, Zhang J-R, Jia Z-Y, Li X-L, Li F, Chen L, Li T, Gong Q-Y. Localization of cerebral functional deficits in treatment-naive, first-episode schizophrenia using resting-state fMRI. NeuroImage. 2010; 49:2901-2906. [PubMed: 19963069] 
Jayakumar PN, Venkatasubramanian G, Keshavan MS, Srinivas JS, Gangadhar BN. MRI volumetric and 31P MRS metabolic correlates of caudate nucleus in antipsychotic-naïve schizophrenia. Acta Psychiatrica Scandinavica. 2006; 114:346-351. [PubMed: 17022794]

Jones P, Rodgers B, Murray R, Marmot M. Child Developmental Risk-Factors for Adult Schizophrenia in the British 1946 Birth Cohort. Lancet. 1994; 344:1398-1402. [PubMed: 7968076]

Kegeles LS, Abi-Dargham A, Frankle WG, Gil R, Cooper TB, Slifstein M, Hwang D-R, Huang Y, Haber SN, Laruelle M. Increased Synaptic Dopamine Function in Associative Regions of the Striatum in Schizophrenia. Arch Gen Psychiatry. 2010; 67:231-239. [PubMed: 20194823]

Kendler KS, McGuire M, Gruenberg AM, O'Hare A, Spellman M, Walsh D. The Roscommon Family Study: I. Methods, Diagnosis of Probands, and Risk of Schizophrenia in Relatives. Arch Gen Psychiatry. 1993; 50:527-540. [PubMed: 8317947]

Langen M, Schnack HG, Nederveen H, Bos D, Lahuis BE, de Jonge MV, van Engeland H, Durston S. Changes in the Developmental Trajectories of Striatum in Autism. Biological Psychiatry. 2009; 66:327-333. [PubMed: 19423078]

Lawrie SM, Whalley HC, Abukmeil SS, Kestelman JN, Donnelly L, Miller P, Best JJK, Owens DGC, Johnstone EC. Brain structure, genetic liability, and psychotic symptoms in subjects at high risk of developing schizophrenia. Biological Psychiatry. 2001; 49:811-823. [PubMed: 11343678]

Lenroot RK, Gogtay N, Greenstein DK, Wells EM, Wallace GL, Clasen LS, Blumenthal JD, Lerch J, Zijdenbos AP, Evans AC, Thompson PM, Giedd JN. Sexual dimorphism of brain developmental trajectories during childhood and adolescence. NeuroImage. 2007; 36:1065-1073. [PubMed: 17513132]

Li H, Chan RCK, McAlonan GM, Gong Q-y. Facial Emotion Processing in Schizophrenia: A Metaanalysis of Functional Neuroimaging Data. Schizophrenia Bulletin. 2010; 36:1029-1039. [PubMed: 19336391]

Lodge DJ, Grace AA. Developmental pathology, dopamine, stress and schizophrenia. International Journal of Developmental Neuroscience. 2011; 29:207-213. [PubMed: 20727962]

Mamah D, Harms MP, Wang L, Barch D, Thompson P, Kim J, Miller MI, Csernansky JG. Basal Ganglia Shape Abnormalities in the Unaffected Siblings of Schizophrenia Patients. Biological Psychiatry. 2008; 64:111-120. [PubMed: 18295189]

Mattai A, Hosanagar A, Weisinger B, Greenstein D, Stidd R, Clasen L, Lalonde F, Rapoport J, Gogtay N. Hippocampal Volume Development in Healthy Siblings of Childhood-Onset Schizophrenia Patients. Am J Psychiatry. 2011; 168:427-435. [PubMed: 21245087]

Maxwell ME. Family Interview for Genetic Studies: Clinical Neurogenetics Branch, Intramural Research Program. NIMH. 1996

Menon V, Anagnoson RT, Glover GH, Pfefferbaum A. Functional Magnetic Resonance Imaging Evidence for Disrupted Basal Ganglia Function in Schizophrenia. Am J Psychiatry. 2001; 158:646-649. [PubMed: 11282705]

Miller TJ, McGlashan TH, Woods SW, Stein K, Driesen N, Corcoran CM, Hoffman R, Davidson L. Symptom Assessment in Schizophrenic Prodromal States. Psychiatric Quarterly. 1999; 70:273287. [PubMed: 10587984]

Morey RA, Inan S, Mitchell TV, Perkins DO, Lieberman JA, Belger A. Imaging Frontostriatal Function in Ultra-High-Risk, Early, and Chronic Schizophrenia During Executive Processing. Arch Gen Psychiatry. 2005; 62:254-262. [PubMed: 15753238]

Müller JL, Röder C, Schuierer G, Klein HE. Subcortical overactivation in untreated schizophrenic patients: A functional magnetic resonance image finger-tapping study. Psychiatry and Clinical Neurosciences. 2002; 56:77-84. [PubMed: 11929574]

Ostby Y, Tamnes CK, Fjell AM, Westlye LT, Due-Tonnessen P, Walhovd KB. Heterogeneity in Subcortical Brain Development: A Structural Magnetic Resonance Imaging Study of Brain Maturation from 8 to 30. Years. J. Neurosci. 2009; 29:11772-11782.

Paus T, Keshavan M, Giedd JN. Why do many psychiatric disorders emerge during adolescence? Nat Rev Neurosci. 2008; 9:947-957. [PubMed: 19002191] 
Prastawa M, Bullitt E, Moon N, Van Leemput K, Gerig G. Automatic brain tumor segmentation by subject specific modification of atlas priors. Academic Radiology. 2003; 10:1341-1348. [PubMed: 14697002]

Rajarethinam R, Upadhyaya A, Tsou P, Upadhyaya M, Keshavan MS. Caudate volume in offspring of patients with schizophrenia. The British Journal of Psychiatry. 2007; 191:258-259. [PubMed: 17766768]

Rapoport JL, Gogtay N. Brain Neuroplasticity in Healthy, Hyperactive and Psychotic Children: Insights from Neuroimaging. Neuropsychopharmacology. 2008; 33:181-197. [PubMed: 17851542]

Rapoport JL, Gogtay N. Childhood onset schizophrenia: support for a progressive neurodevelopmental disorder. International Journal of Developmental Neuroscience. 2011; 29:251-258. [PubMed: 20955775]

Shaw P, Gogtay N, Rapoport J. Childhood psychiatric disorders as anomalies in neurodevelopmental trajectories. Human Brain Mapping. 2010; 31:917-925. [PubMed: 20496382]

Shenton ME, Dickey CC, Frumin M, McCarley RW. A review of MRI findings in schizophrenia. Schizophrenia Research. 2001; 49:1-52. [PubMed: 11343862]

Tienari P, Wynne LC, Laksy K, Moring J, Nieminen P, Sorri A, Lahti I, Wahlberg K-E. Genetic Boundaries of the Schizophrenia Spectrum: Evidence From the Finnish Adoptive Family Study of Schizophrenia. Am J Psychiatry. 2003; 160:1587-1594. [PubMed: 12944332]

van Erp TGM, Therman S, Pirkola T, Tuulio-Henriksson A, Glahn DC, Bachman P, Huttunen MO, Lönnqvist J, Hietanen M, Kaprio J, Koskenvuo M, Cannon TD. Verbal recall and recognition in twins discordant for schizophrenia. Psychiatry Research. 2008; 159:271-280. [PubMed: 18442861]

Velakoulis D, Wood SJ, Wong MTH, McGorry PD, Yung A, Phillips L, Smith D, Brewer W, Proffitt T, Desmond P, Pantelis C. Hippocampal and Amygdala Volumes According to Psychosis Stage and Diagnosis: A Magnetic Resonance Imaging Study of Chronic Schizophrenia, First-Episode Psychosis, and Ultra-High-Risk Individuals. Arch Gen Psychiatry. 2006; 63:139-149. [PubMed: 16461856]

Vita A, De Peri L, Silenzi C, Dieci M. Brain morphology in first-episode schizophrenia: A metaanalysis of quantitative magnetic resonance imaging studies. Schizophrenia Research. 2006; 82:75-88. [PubMed: 16377156] 

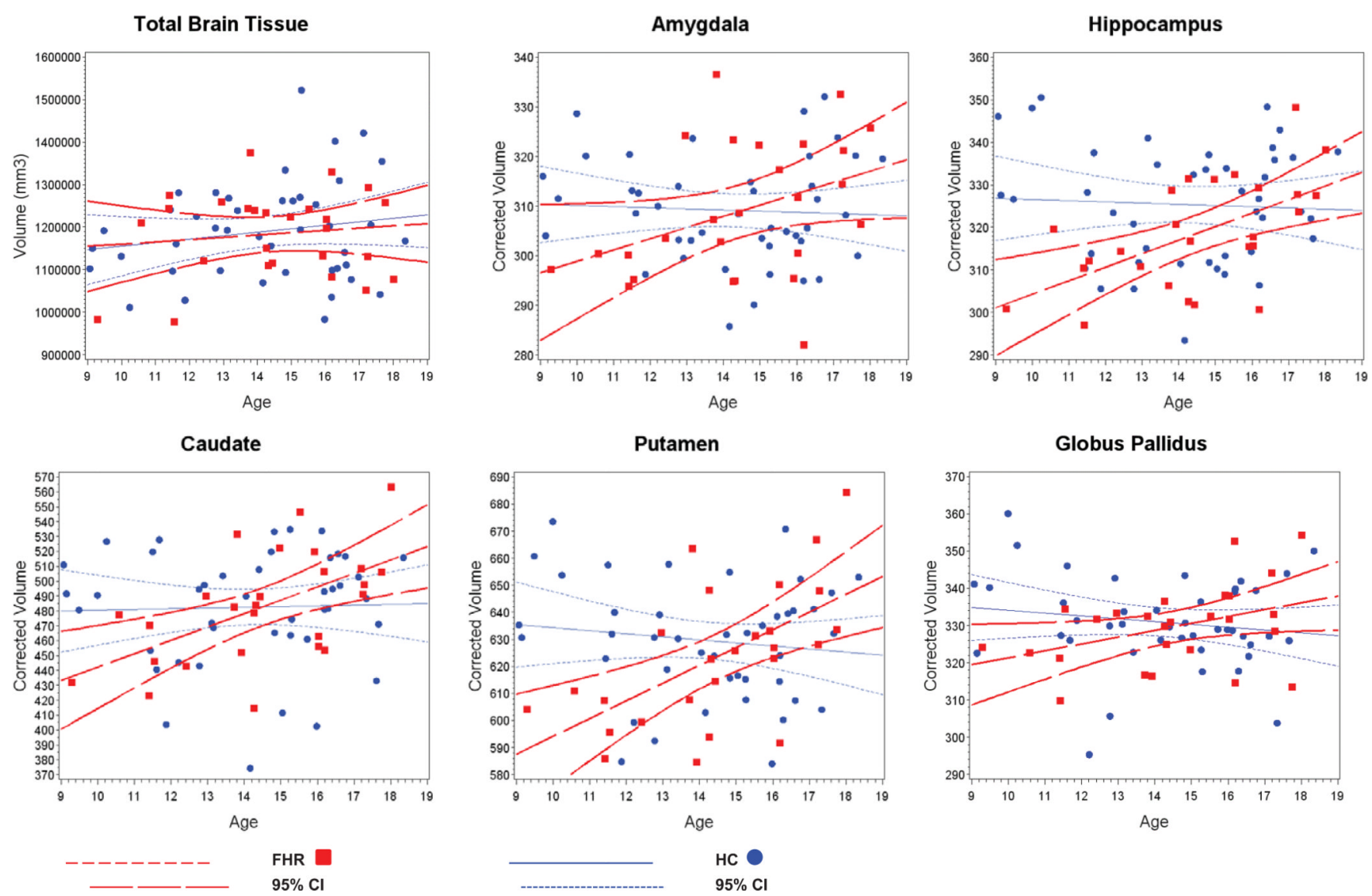

Figure 1.

Age and group differences of subcortical structures and total brain tissue. FHR=familial high risk; $\mathrm{HC}=$ healthy comparison; $\mathrm{CI}=$ confidence interval. 


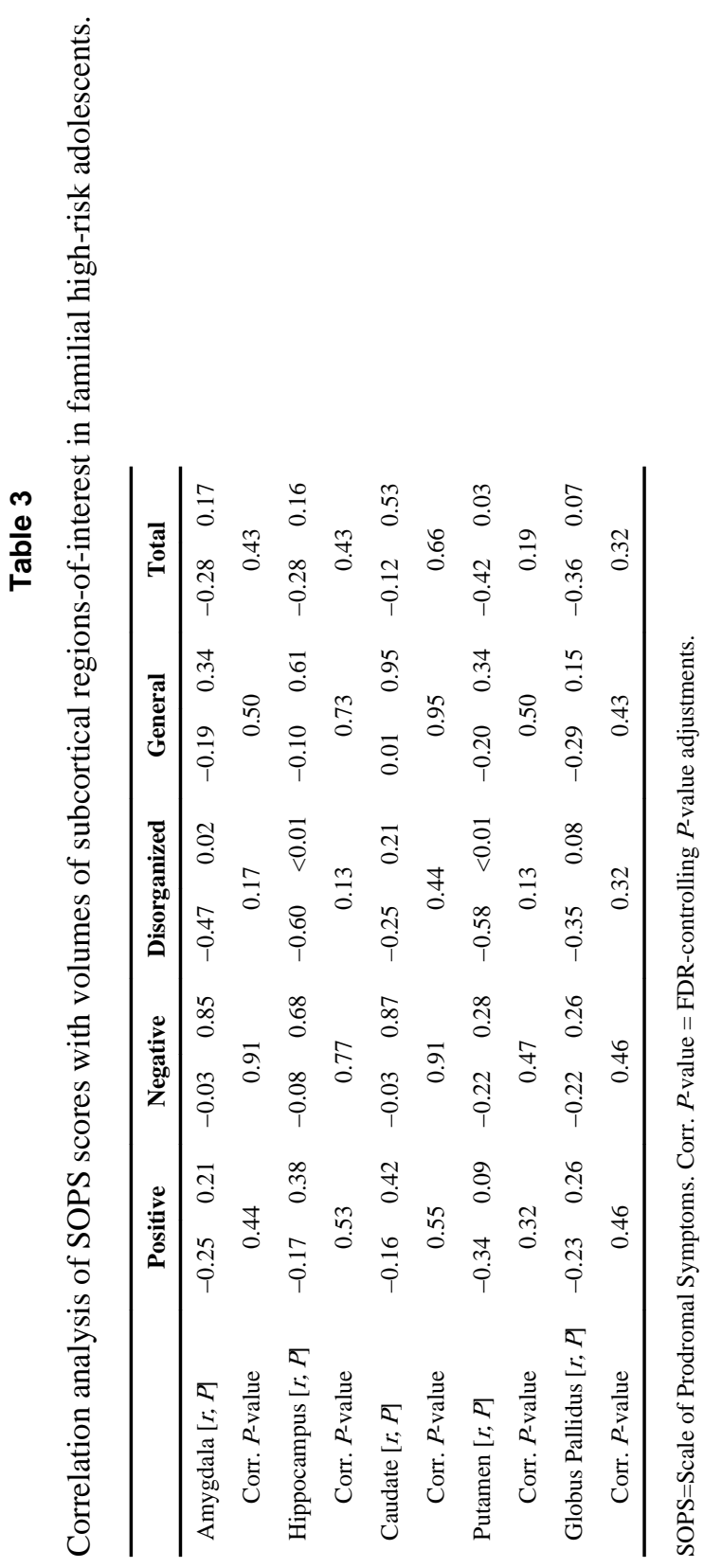

